\title{
ADOÇÃO E USO DE PORTAIS DE GOVERNO ELETRÔNICO NO AMBIENTE DO SISTEMA NACIONAL DE CIÊNCIA E TECNOLOGIA E INOVAÇÃO um estudo baseado no modelo UTAUT
}

\section{1- Érico da Silva Costa*}

Mestre em Administração de Empresas pela Universidade Metodista de São Paulo (UMESP), Brasil. Professor do Instituto Federal de Educação, Ciência e Tecnologia de São Paulo (IFSP), Brasil. adm_erico@hotmail.com http://lattes.cnpq.br/3348528532596825

\section{2- Dagmar Silva Pinto de Castro}

Doutora em Psicologia pela Universidade de São Paulo (USP), Brasil.

Professora do Programa de Pós-Graduação em Administração da Universidade Metodista de Piracicaba (UNIMEP), Brasil. dscastro@unimep.br

http://lattes.cnpq.br/6371527386043638

\section{3- Alexandre Cappellozza}

Doutor em Administração de Empresas pela Fundação Getúlio Vargas (EAESP/FGV), Brasil. Professor do Programa de Pós-Graduação em Administração da Universidade Metodista de São Paulo (UMESP), Brasil. alexandre.cappellozza@metodista.br http://lattes.cnpq.br/8358857348226421

\begin{tabular}{l} 
Diego Maganhotto Coraiola - Editor Geral \\
\multicolumn{1}{c}{ Editor responsável pela submissão: } \\
Emerson Maccari. \\
Artigo analisado via processo de revisão duplo cego (Double-blind). \\
Recebido em: $18 / 02 / 2014$ \\
Aprovado em: $31 / 08 / 2014$ \\
Última Alteração: $14 / 09 / 2014$
\end{tabular}

* Contato Principal: Av. Rio Grande do Norte, 450. Caraguatatuba - SP, Brasil. CEP: 11665-310.. 


\title{
ADOÇÃO E USO DE PORTAIS DE GOVERNO ELETRÔNICO NO AMBIENTE DO SISTEMA NACIONAL DE CIÊNCIA E TECNOLOGIA E INOVAÇÃO: UM ESTUDO BASEADO NO MODELO UTAUT
}

\begin{abstract}
As relações entre cidadãos e governos têm sido complementadas pelo uso das Tecnologias da Informação e Comunicação (TICS), sobretudo, pela preferência por serviços transacionais on-line em ambientes virtuais, associada à conveniência aos usuários. O Portal Inovação visa atender às demandas do Sistema Nacional de Ciência, Tecnologia e Inovação (SNCTI) do País e, sobretudo, auxiliar os usuários na coleta e transmissão de informações. Esta pesquisa analisa elementos da aceitação do Portal Inovação, identificando os fatores preditivos da adoção desta tecnologia pelos seus usuários. Para atingir os objetivos propostos, recorreu-se à Teoria Unificada de Adoção de Tecnologia (UTAUT) para análise das percepções dos usuários do portal. Os principais resultados obtidos demonstraram alta magnitude e significância preditiva sobre a Intenção Comportamental de Uso do Portal pelos fatores: Expectativa de Desempenho e Influência Social. A conclusão principal do presente estudo é a de que ao considerarmos a aceitação de um portal governamental em que a adoção é voluntária, o fator social é altamente influente na intenção de uso da tecnologia, bem como os aspectos relacionados à produtividade consequente do usuário e o senso de utilidade; além da facilidade de interação e domínio da ferramenta.
\end{abstract}

\section{Keywords}

Teoria Unificada de Aceitação e Uso da Tecnologia (UTAUT). Adoção de tecnologia. Portal Inovação. governo eletrônico. gestão da inovação.

\section{ADOPTION AND USE OF ELETRONIC GOVERNMENT WEBSITES IN THE NATIONAL SCIENCE AND TECHNOLOGY AND INNOVATION ENVIRONMENT: A STUDY BASED ON THE UTAUT MODEL}

\section{RESUMO}

The relationships between citizens and governments have been complemented by the intensive use of Information Technology and Communication (ICT) for transactional services online. The Brazilian Innovation Portal was developed by the Ministry of Science, Technology and Innovation (MSTI) in partnership with the Center for Strategic Studies and Management (CSSM), Brazilian Association for Industrial Development (BAID) and Instituto Stela to meet demands the National System of Science, Technology and Innovation (NSSTI). Based on Unified Theory of Acceptance and Use of Technology and structural equation modeling technique, the main results of this study showed high predictive significance and magnitude of the Behavioral Intention of Use Portal by the following factors: Expected Performance and Social Influence. Therefore, the social factor is highly influential in the intention to use the technology as well as aspects related to user productivity and the consequent sense utility, plus the ease of interaction and domain tool. Such findings supplement new perspectives in studies in the field of e-Gov, as well as in evaluation of government projects.

\section{Palavras-Chave}

Unified Theory of Acceptance and Use of Technology (UTAUT). Technology Adoption. Innovation Portal. e-Government. 


\section{Introduction}

Presenciamos nas últimas décadas, uma expansão considerável das Tecnologias de Informação e Comunicação (TICS), que têm provocado uma nova dinâmica nas relações com o Estado, o que vem contribuindo para uma expansão quantitativa dos serviços públicos.

Neste contexto, as relações entre cidadãos e governos têm sido fortemente impactadas pelo intenso uso das Tecnologias da Informação e Comunicação (TICs) pelos cidadãos e empresas, sobretudo, pela preferência por serviços transacionais on-line em ambientes virtuais, associada à conveniência proporcionada aos usuários (Barbosa, 2008).

No entanto, as transformações inseridas pelas TICs impõem uma nova realidade à forma de legislar e de administração pública porque criou o potencial para a existência de fluxos de informação bidirecionais, tornando possível que a sociedade civil influencie o Estado sobre princípios democráticos, sem paralisar necessariamente sua efetividade como agência de interesse público (Castells, 2000).

A implantação e o desenvolvimento de programas de governo eletrônico (e-Gov) em vários países são uma das aplicações notáveis da rápida e intensa adoção das TICs em organizações públicas e que aprimoram a forma como o governo gerencia o relacionamento entre os órgãos prestadores de serviços públicos e os cidadãos (McNeal et al., 2003). No Brasil, a implantação desses programas costuma atingir todos os níveis de governo (federal, estadual e municipal), que pode favorecer o aumento do desempenho e da eficiência da administração pública, bem como fomentar o desenvolvimento de processos e o compartilhamento de informações entre as diferentes esferas governamentais (Cetic.BR, 2011).

Em meados do ano de 2004, o Ministério da Ciência, Tecnologia e Inovação (MCTI), após a implantação do marco regulatório da Inovação (Lei n- 10.973/2004), demanda o desenvolvimento de um instrumento que proporcionasse um espaço de colaboração para a cooperação tecnológica e que vinculasse novas tecnologias da comunicação e informação. Em decorrência dessa solicitação, foi desenvolvido um projeto multi-institucional, coordenado pelo Centro de Gestão e Estudos Estratégicos (CGEE) em cooperação com consultores de inovação, com a Agência Brasileira de Desenvolvimento Industrial (ABDI), responsável por sua gestão operacional e com o Instituto Stela, instituição de $P \& D$, o desenvolvimento do Portal Inovação.

A criação do Portal Inovação (www.portalinovacao.mct.gov.br) é uma resposta do Ministério da Ciência, Tecnologia e Inovação (MCTI) às necessidades de aperfeiçoamento do Sistema Nacional de Ciência, Tecnologia e Inovação (SNCTI) de contar com um instrumento de apoio à cooperação tecnológica que aproxime os atores de inovação do País, tais como profissionais, organizações, instituições científicas, unidades institucionais de pesquisa e desenvolvimento e fomente o desenvolvimento econômico e industrial.

A relevância conferida à política de Ciência, Tecnologia e Inovação no processo de desenvolvimento do País decorre do reconhecimento dos órgãos federais sobre os possíveis impactos positivos das inovações científicas e tecnológicas à economia nacional, à preservação dos recursos naturais e a manutenção e melhoria do padrão de vida da população brasileira a partir da incorporação destas inovações aos processos gerenciais e produtivos existentes (Brasil, 2011).

Contudo, apenas a expansão quantitativa de sistemas de informação, sem uma perspectiva de melhoria contínua, oferta de serviços integrados que privilegiem a transparência, eficiência, acessibilidade e aceitação do sistema pelos interessados pode reduzir o êxito dos projetos de governo eletrônico (Correa, 2011).

Assim, compreender como as variáveis e aspectos decorrentes da introdução de novas tecnologias da informação e da comunicação interferem na administração pública se revela como uma possibilidade de estudos no campo de gestão dos sistemas de informação. Desta forma, estudos que explorem percepções dos usuários sobre os sistemas de tecnologia governamentais podem ser realizados com o objetivo de identificar se tais ações ou projetos estão atingindo plenamente às pretensões planejadas; bem como prestar contas à própria sociedade que os utiliza sobre a real efetividade destes.

Este trabalho objetiva analisar a aceitação do Portal Inovação identificando os fatores preditivos da intenção comportamental de uso e do comportamento de uso direcionadores da adoção da tecnologia por seus usuários e se justifica pela relevância do tema no cenário nacional e internacional, inclusive, pela oportunidade de pesquisa sobre as temáticas: políticas públicas, governo eletrônico e adoção de tecnologia no contexto de participação e engajamento acadêmico e social. 
Para tanto, o artigo está estruturado em quatro seções. Após essa introdução são abordados, no referencial teórico os seguintes temas centrais: os conceitos de governo eletrônico, informações sobre Sistema Nacional de Ciência, Tecnologia e Inovação, além da Teoria Unificada de Aceitação e Uso da Tecnologia. A terceira seção aborda o percurso metodológico utilizado na pesquisa que contempla a descrição e análise dos dados. Finalmente, são apresentadas as conclusões do estudo.

\section{Referencial Teórico}

O referencial teórico deste estudo está dividido em três partes principais, quais sejam: o governo eletrônico, Sistema Nacional de Ciência, Tecnologia e Inovação e a Teoria Unificada de Aceitação e Uso da Tecnologia.

\subsection{O Governo Eletrônico (E-Gov)}

O avanço das TICs possibilitou inúmeros benefícios à sociedade e o desenvolvimento de uma extensa gama de serviços oferecidos na esfera governamental, tais como, a disponibilidade de serviços públicos em portais eletrônicos destinados a atender as necessidades dos cidadãos.

Diversos estudos definem o governo eletrônico sob múltiplas formas: Maciel (2008) afirma que as estratégias e ações governamentais para uso dos recursos das TICS com a intenção de modernizar a máquina administrativa e atender as necessidades do cidadão-contribuinte definem este conceito. Assim, o emprego dos recursos tecnológicos para otimizar ou aperfeiçoar o método pelo qual cidadãos, parceiros, servidores e outros participantes do governo possam interagir e realizar suas atribuições públicas caracterizam o e-Gov (Koh, Ryan, \& Prybutok, 2005). Para Osborne (1997), e-Gov se refere à modernização da administração pública, com procedimentos operacionais e administrativos mais eficientes, além da utilização dos recursos das TiCs para estes fins (Agune \& Carlos, 2005; Osborne, 1997).

As ações de e-Gov visam não só a inclusão digital dos cidadãos para promover a universalização do acesso, mas também à inclusão social pela uniformização do acesso ao conhecimento sobre leis e serviços disponíveis à sociedade, bem como geram oportunidades para a redução dos custos de produção e de uso dos serviços. Dessa forma, acabam por otimizar a infraestrutura administrativa e requerer melhorias expressivas em atendimentos e capacitação de servidores por meio de uso de novas tecnologias (Correa, 2011).

Neste sentido, o desenvolvimento de portais eletrônicos que disponibilizam serviços governamentais, entre outras funcionalidades, promove a utilização da TICs orientadas à democratização do acesso à informação e aprimoramento da prestação de serviços públicos pelo aumento da eficiência e eficácia das atividades governamentais, aprimorando as relações entre o governo, os cidadãos, as organizações e órgãos do próprio governo (Prado, Ramalho, Souza, Cunha, \& Reinhard, 2011).

A partir da popularização do uso da Internet e o respectivo desenvolvimento de aplicações orientadas a este ambiente virtual, observou-se uma crescente proliferação da oferta de serviços públicos no mundo baseadas nesta plataforma e diversos países disponibilizaram aplicações eGovernment, tais como, os Estados Unidos e boa parte da Europa. Entre outras aplicações, o Brasil ocupa lugar de destaque na cobrança eletrônica de impostos, além do desenvolvimento de sistemas de informação dedicados a Ciência e Tecnologia, tal como, o Sistema Nacional de Ciência, Tecnologia e Inovação (SNCTI) (Aldricha, Bertota, \& McClurea, 2002; Damian \& Merlo, 2013).

\subsection{Sistema Nacional de Ciência, Tecnologia e Inovação (SNCTI)}

O Sistema Nacional de Inovação pode ser definido como um conjunto de instituições, atores e mecanismos em um país que contribui para a criação, o avanço e desenvolvimento das inovações tecnológicas a serem difundidas pelos institutos de pesquisa e seus laboratórios de desenvolvimento, instituições, sistema educacional, firmas, agências governamentais, departamentos de legislação de propriedade intelectual e as universidades (Freeman, 1995).

A Organização para a Cooperação e Desenvolvimento Econômico (OCDE) amplia tal entendimento ao caracterizar como um fenômeno político, complexo e sistêmico de ampla abrangência em que o conjunto de instituições e organizações responsável pela criação e adoção de inovações em um determinado país conduz políticas nacionais que enfatizam as interações entre 
instituições que participam do amplo processo de criação do conhecimento e da sua difusão (OCDE, 1996).

No escopo dessa questão, o Portal Inovação desempenha um papel relevante para o cenário do SNCTI do País, pois se configura em um espaço de interação, cooperação tecnológica e apoio à tomada de decisão para os diversos atores do sistema brasileiro de inovação.

\subsubsection{O Portal Inovação (MCTI)}

A demanda pela criação do Portal Inovação se inicia em meados do ano de 2004, quando o MCT, durante a criação e o desenvolvimento do marco regulatório - Lei no 10.973/2004, articula uma série de ações com vistas a consolidar um instrumento de aproximação entre universidade, governo e indústria.

Tal proposta tinha como referenciais a experiência americana, como o Ato Bayh-Dole ${ }^{1}$ e a lei francesa de inovação, bem como iniciativas nacionais que criaram a base conceitual para o desenvolvimento do Portal Inovação - a Plataforma Lattes (CNPQ, 1998-2004) e o Projeto Inventec (SCT-BA). Ambos os projetos foram os precursores do Portal Inovação, pois já consolidavam experiências em ciência e tecnologia, que serviram de base conceitual para o desenvolvimento do projeto. O Portal Lattes, já em 2004, possuía uma base com cerca de 460 mil currículos e, portanto, configurava-se em um excelente repositório com informações acadêmicas e de competências técnicas.

\begin{tabular}{|c|c|}
\hline Funcionalidade & Descrição \\
\hline Agentes de inovação & $\begin{array}{l}\text { Nesse ambiente, o governo e as entidades representativas localizam ofertantes e demandantes, } \\
\text { acessam indicadores e identificam as dinâmicas das cooperações e do processo de inovação } \\
\text { nacional. }\end{array}$ \\
\hline $\begin{array}{l}\text { Buscas por Competências e } \\
\text { Oportunidades }\end{array}$ & $\begin{array}{l}\text { Empregam sistemas de conhecimentos dimensionados para localizar especialistas nas diversas } \\
\text { áreas técnico-científicas, além de oportunidades e cooperação técnico-científica nos vários } \\
\text { setores sócioeconômicos, provenientes das demandas inseridas na platafor }\end{array}$ \\
\hline Empresas & $\begin{array}{l}\text { Permite localizar e ofertar competências e oportunidades em todas as áreas do conhecimento e } \\
\text { em diferentes setores da economia, possibilitando acesso a competências; interação com } \\
\text { especialistas; obter informações exclusivas; além de permitir indicar deman }\end{array}$ \\
\hline Especialistas e Grupos de P\&D & $\begin{array}{l}\text { Permite a oferta de competências e localização de oportunidades de interação com o setor } \\
\text { empresarial. }\end{array}$ \\
\hline ICTIs & $\begin{array}{l}\text { Ambiente em que os institutos gerenciam o processo de cooperação e localizam instrumentos } \\
\text { facilitadores para o cumprimento dos quesitos da Lei de Inovação, bem como permite aos } \\
\text { dirigentes de instituições de } P \& D \text { interagir com empresas e com a comunidade té }\end{array}$ \\
\hline Indicadores Gerais & $\begin{array}{l}\text { Possibilitam o acompanhamento de índices regionalizados de uso do Portal (competências, } \\
\text { oportunidades e interação entre os atores); }\end{array}$ \\
\hline Informações Estratégicas & $\begin{array}{l}\text { Os participantes (empresas, especialistas e dirigentes de ICTIs) em ambientes individualizados, } \\
\text { localizam informações estratégicas que permitem a geração de gráficos sobre relacionamento } \\
\text { entre os profissionais vinculados à Plataforma Lattes (currículo); }\end{array}$ \\
\hline Interação e Cooperação & $\begin{array}{l}\text { Permite a empresas e especialistas salvar buscas realizadas no Portal, bem como iniciar a } \\
\text { interação por intermédio de envio de propostas aos cadastrados na plataforma; }\end{array}$ \\
\hline Público em Geral & $\begin{array}{l}\text { Demais interessados podem participar do Portal Inovação, beneficiando-se do conteúdo } \\
\text { disponibilizado que permite ao usuário o acesso a indicadores regionais, localizar pesquisadores } \\
\text { nos diversos domínios técnico-científicos por competência; buscar oportun }\end{array}$ \\
\hline Sistemas d & $\begin{array}{l}\text { O Portal Inovação congrega sistemas avançados que permitem subsidiar a interação e a } \\
\text { cooperação entre empresas e comunidade técnico-científica. Destacando-se, os mecanismos de } \\
\text { busca criativa, baseada em coocorrências, sistemas de análise de redes sociais }\end{array}$ \\
\hline
\end{tabular}

Figura 1 - Funcionalidades do Portal Inovação

Nota. Fonte: elaborado pelos autores.

O Portal Inovação é uma resposta do MCT à necessidade do Sistema Nacional de Ciência e Tecnologia contar com um instrumento de apoio à cooperação tecnológica que aproxime os atores 
de inovação do País. Trata-se de um projeto multiinstitucional que foi desenvolvido sobre a coordenação do CGEE em cooperação com consultores de inovação, com a ABDI, responsável por sua gestão operacional, e com o Instituto Stela, instituição de P\&D que desenvolveu a arquitetura do Portal Inovação.

No Portal Inovação, a cooperação é promovida por intermédio de diferentes recursos de informação em que, de um lado, estão às fontes de informação sobre as competências nacionais (oriundas das indexações de currículos e de grupos de pesquisa do SNCTI) e, de outro, as oportunidades de cooperação técnico-científica (resultado da declaração de demandas por parte de empresas).

Entre outros recursos, o Portal Inovação concentra um conjunto abrangente de serviços e produtos destinados à promoção da interação, cooperação e busca de informações relacionadas às competências e oportunidades apresentadas na plataforma, de acordo com as funcionalidades descritas na Figura 1.

Ressalta-se que o principal diferencial do Portal Inovação se refere às conjugações das bases de gestão que possibilitaram a formação do diretório nacional de competências em C\&T, além dos recursos de busca e geração de novas informações em inovação das empresas e comunidade técnico-científica (via sistemas componentes do Portal) e no conjunto de sistemas projetados para gerenciar as atividades de interação e de conhecimento sobre o processo de inovação.

Dada a disponibilidade de diversas funcionalidades aos usuários, a avaliação dos serviços prestados a sociedade representa, também, uma forma de avaliar as diretrizes e políticas governamentais instituídas pelo Estado. Neste aspecto, o desenvolvimento e implantação de projetos de governo eletrônico devem contemplar mecanismos que mensurem a adoção, aderência e utilização da tecnologia pelos usuários e potenciais usuários.

\subsection{Teoria Unificada de Aceitação e Uso da Tecnologia (UTAUT)}

A adoção individual de Tecnologia da Informação (TI) tem sido estudada por diferentes e importantes modelos conceituais: Modelo de Aceitação da Tecnologia (TAM) - Technology Acceptance Model (Davis, Bagozzi, \& Warshaw, 1989), Extensão do Modelo de Aceitação de Tecnologia (TAM2) - Extension Technology Acceptance Model (Venkatesh \& Davis, 2000) que, em geral, emanaram de teorias da Psicologia como a Teoria da Ação Racional (TRA) - Theory of Reasoned Action, elaborada por Fishbein e Ajzen (1975), e a Teoria do Comportamento Planejado (TPB) - Theory of Planned Behavior, desenvolvida por Ajzen e Fishbein, em 1985, derivada da TRA.

Um importante modelo de análise proveniente destas citações, resultado da minuciosa análise de um total de oito modelos anteriores, é o Modelo Unificado de Aceitação de Tecnologia (UTAUT) Unified Theory of Acceptance and Use of Technology, de Venkatesh, Morris, Davis e Davis (2003), que tem sido largamente analisado e validado em vários cenários de tecnologia e ambientes.

A elaboração do modelo teórico UTAUT é resultante da multiplicidade de diversos modelos para analisar a aceitação de tecnologia da informação e pode ser utilizado para a avaliação da probabilidade de sucesso de uma nova tecnologia a ser implantada por meio da análise da aceitação do potencial usuário do SI. O modelo foi sugerido com o intuito de prover um instrumento para gestores com necessidade de ponderar a probabilidade de sucesso da introdução de novas tecnologias e ajudá-los a entender quais são os direcionadores de sua aceitação, além de explicitar as intenções do usuário para utilizar um determinando sistema de informação e comportamento de uso subsequente (Venkatesh et al., 2003).

Além disto, este é um modelo teórico que possibilita a realização de estudos científicos com diferentes tipos de tecnologia, usuários e ambientes de utilização, tais como, ferramentas de ensino a distância (Al-Gahtani, 2001), aplicativos de escritório (Chiu \& Wang, 2008) e voto eletrônico (Yurong \& Murphy, 2007), entre outras possibilidades. Em suma, nota-se que os estudos que aplicam o modelo UTAUT ao estudo das TIC's objetivam a mensuração das percepções dos usuários quanto à adoção de uma determinada tecnologia de informação por meio das variáveis que compreendem o modelo preconizado por Venkatesh et al. (2003).

A Teoria Unificada de Aceitação e Uso da Tecnologia está sustentada em quatro construtos: "Expectativa de Desempenho"; "Expectativa de Esforço"; "Influência Social" e "Condições Facilitadoras" que são determinantes diretos da intenção e comportamento de uso da tecnologia de informação analisada. 
Gênero, Idade, Experiência e Voluntariedade de uso são variáveis que moderam sobre os quatro principais construtos associados com a intenção de uso e comportamento da aplicação de acordo com a Figura 2 (Venkatesh et al., 2003).

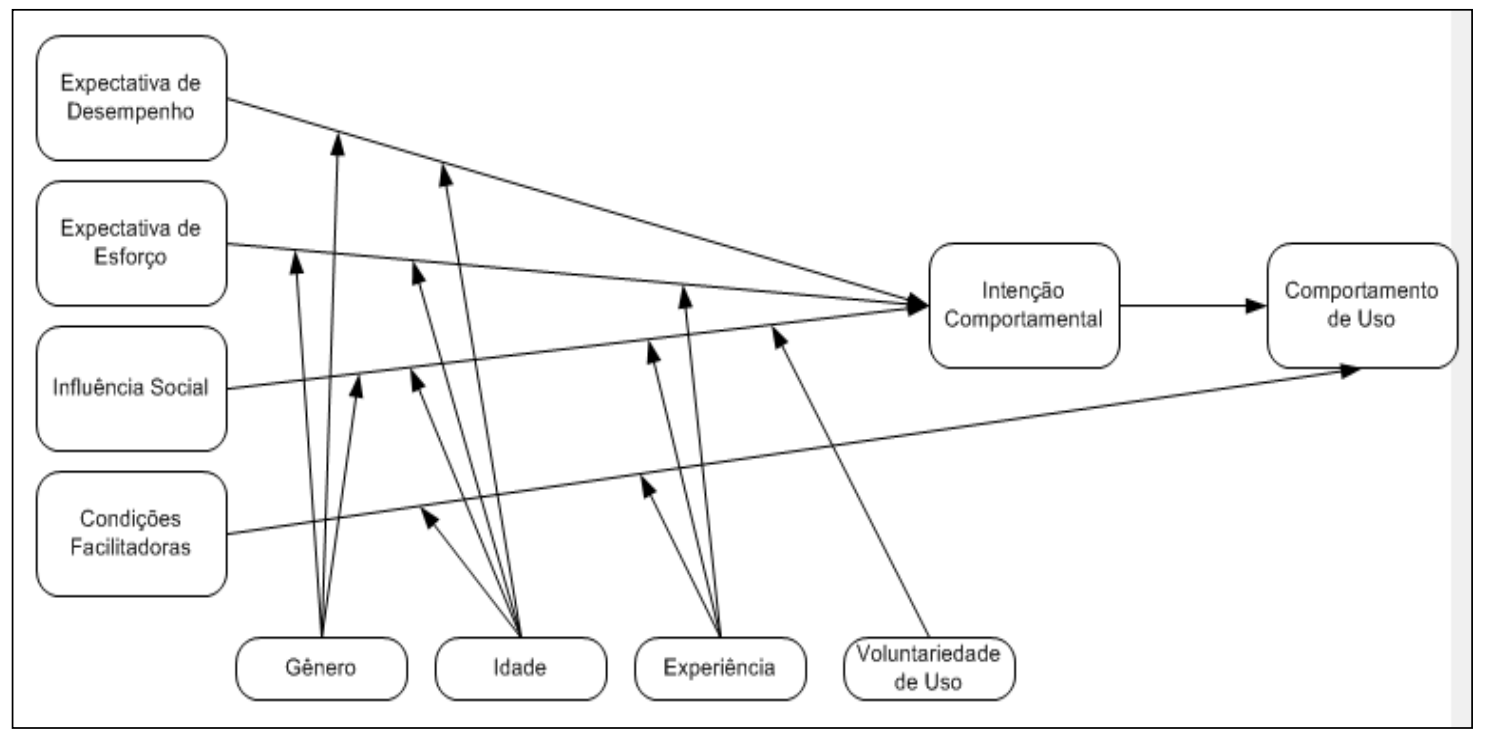

Figura 2 - Teoria Unificada de Aceitação e Uso da Tecnologia (UTAUT)

Nota. Fonte: Adaptado de Venkatesh et al. (2003)

O construto "Expectativa de Desempenho" desenvolvido por Venkatesh et al. (2003) mensura o nível em que o indivíduo acredita que a utilização do sistema possa ajudá-lo a obter ganhos de desempenho em suas atribuições profissionais. Já o construto "Expectativa de Esforço" está relacionado com a percepção do usuário sobre a facilidade de utilização do sistema (Venkatesh et al., 2003).

O construto "Influência Social" avalia a percepção do usuário quanto à utilização do sistema sob a influência do ambiente social que o usuário está inserido, além de percepções associadas à imagem, ou status, que o indivíduo adquire pelo uso da aplicação. O construto "Condições Facilitadoras" indica o nível em que o indivíduo acredita que a organização e a infraestrutura existentes suportam a utilização do sistema (Venkatesh et al., 2003).

Fundamentado na Teoria de Ação Racional de Fishbein e Azjen (1975), o construto "Intenção Comportamental de Uso" no modelo UTAUT pode ser definido como um propósito, deliberação, desejo ou vontade de utilização da tecnologia analisada.

Neste sentido, a partir dos construtos e seus respectivos relacionamentos contidos no modelo UTAUT, elaboram-se as hipóteses a serem testadas sobre a adoção do portal inovação:

H1: A Expectativa de Desempenho influencia a Intenção de Uso do Portal Inovação.

H2: A Expectativa de Esforço influencia a Intenção de Uso do Portal Inovação.

H3: A Influência Social influencia a Intenção de Uso do Portal Inovação.

H4: Condições facilitadoras influenciam a Intenção de Uso do Portal Inovação.

H5: A Intenção de Uso influencia o Comportamento de Uso do Portal Inovação

\section{Procedimentos Metodológicos}

Nesta seção serão apresentadas informações sobre a classificação da pesquisa, dados sobre a amostra do estudo e instrumento, além dos resultados dos testes estatísticos e respectivas validações necessárias à confirmação das hipóteses e avaliação do modelo de mensuração, entre outros aspectos. 


\subsection{Informações do Instrumento de Medida e Amostra}

Classificada como pesquisa quantitativa, este estudo utiliza técnicas estatísticas que analisam dados coletados em questionários que objetivam analisar como o Portal Inovação é aceito por seus usuários identificando os fatores preditivos da Intenção Comportamental de Uso e Comportamento de Uso (Richardson, 1989; Roesch, 1999).

O instrumento de medida é composto por 28 questões e foi empregada uma escala de sete pontos, sendo: 1 (um) para "Discordo totalmente" e 7 (sete) para "Concordo totalmente" e pode ser visualizado na seção "Apêndice" deste estudo.

A coleta de dados foi realizada por intermédio de um questionário eletrônico desenvolvido no ambiente Surveymonkey ${ }^{\circledR}$ (www.surveymonkey.com) e encaminhado por e-mail aos usuários do Portal Inovação.

Foram enviados 12.948 e-mails aos usuários do portal e coletados 280 questionários respondidos, dos quais 16 (dezesseis) foram descartados em decorrência do não preenchimento completo com informações úteis à pesquisa, sendo contemplados para fins de análise 264 questionários.

Para a análise de dados, empregou-se a técnica de modelagem de equações estruturais, comumente utilizada para procedimentos analíticos em que existem situações de cálculo de variáveis dependentes e independentes; além da manipulação de relações concomitantes entre essas variáveis (Hair, Black, Babin, Anderson, \& Tathan, 2009). Para a obtenção dos resultados que envolvem o modelo das equações estruturais, utilizou-se o software SmartPLS 2.0.M3 (Ringle et al, 2005).

A amostra é composta por 264 participantes, dos quais 51\% pertencem ao gênero masculino e $49 \%$ ao feminino, sendo a média de idade verificada equivalente a 42 anos. Quanto ao estado civil dos respondentes $61,7 \%$ são casados, $18,6 \%$ solteiros, $19,7 \%$ responderam como outros. Sobre o setor de atuação, $62,9 \%$ informaram que atuam no setor público, $36,4 \%$ no setor privado, e $0,8 \%$ atuam em empresas de economia mista. Com o objetivo de evitar estratificação da amostra por localização dos respondentes, foram coletados dados em diferentes regiões geográficas de acordo com a Figura 3:

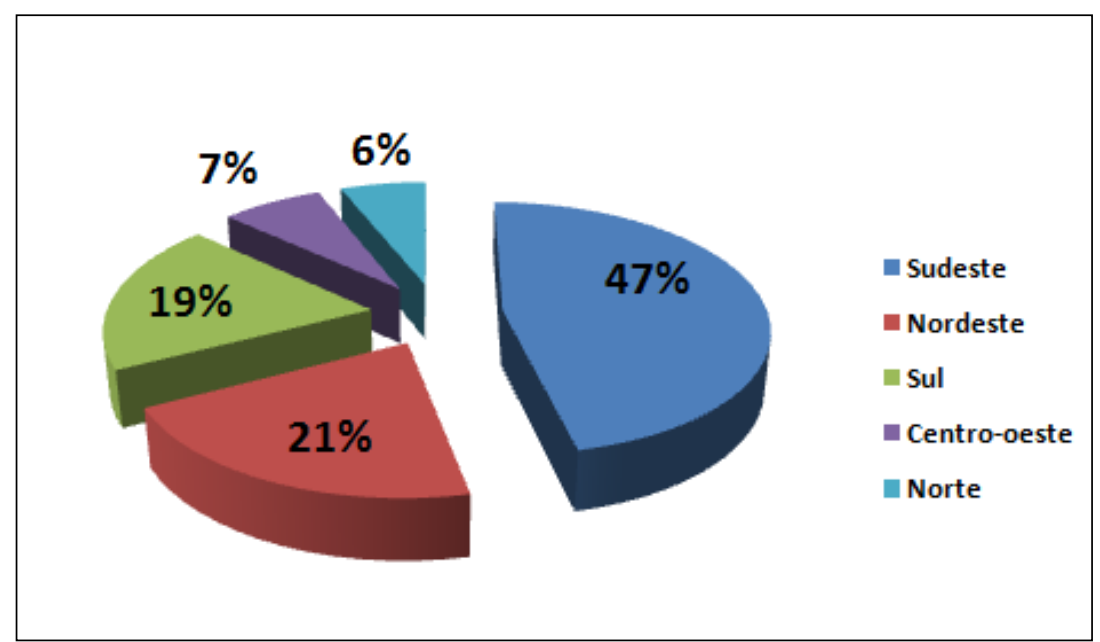

Figura 3 - Distribuição dos respondentes da pesquisa por região geográfica brasileira Nota. Fonte: Elaborado pelos autores.

Quanto ao nível escolar dos respondentes, identificou-se que $64 \%$ possuem pós-graduação stricto sensu, 15,2\% lato sensu, 6,8\% superior incompleto, 4,5\% responderam que possuem livre docência e $2,3 \%$ o segundo grau completo. Sobre a forma de utilização do Portal Inovação, $81,8 \%$ responderam que é voluntária, e 18,9\% declararam que as organizações onde atuam exigem a utilização do Portal no desenvolvimento de suas atribuições.

\subsection{Validade Discriminante e Confiabilidade do Modelo}

Para verificar a validade discriminante e a confiabilidade do modelo UTAUT aplicado ao objeto de pesquisa e assegurar se os conceitos medidos são suficientemente precisos, foi utilizado o critério 
de Fornell-Larker (1981), que estabelece que a raiz quadrada de Variância Média Explicada (AVE) deve ser maior que as intercorrelações entre os demais construtos. Conforme se evidencia na Tabela 1 , os valores dispostos diagonalmente (raiz quadrada da variância extraída do construto) apresentam coeficientes maiores que as demais intercorrelações - o que ratifica que as afirmativas apresentadas aos respondentes mensuram as variáveis do modelo e não se confundem umas com as outras (Hair et al., 2009).

Tabela 1

Correlações entre construtos

\begin{tabular}{lllllll}
\hline & COMP_USO & COND_FAC & EXP_DES & EXP_ESF & INFL_SOC & INT_USO \\
\hline COMP_USO & $\mathbf{1}, 0000$ & & & & & \\
COND_FAC & $-0,3040$ & 0,8418 & & & & \\
EXP_DES & $-0,3193$ & 0,9222 & 0,8472 & & & \\
EXP_ESF & $-0,2636$ & 0,7142 & 0,7285 & 0,9074 & & \\
INFL_SOC & $-0,3550$ & 0,7417 & 0,7757 & 0,7489 & 0,8045 & \\
INT_USO & $-0,2887$ & 0,6012 & 0,6560 & 0,6044 & 0,7400 & $\mathbf{0 , 9 7 1 7}$ \\
\hline
\end{tabular}

Nota. Comp_uso: Comportamento de Uso da Tecnologia, Cond_Fac: Condições Facilitadoras; Exp_Des:Expectativa de Desempenho; Exp_Esf: Expectativa de Esforço; Infi_soc: Influência Social; Int_Uso: Intenção de uso da tecnologia.

Fonte: Elaborado pelos autores.

Para testar a validade discriminante do modelo aplicado, fundamentou-se o teste em análise fatorial confirmatória que, segundo Hair et al. (2009), é significativo na ratificação de escalas para a mensuração de construtos, em que, para fins de demonstração da adequação do construto, a validação deve apresentar as cargas dos indicadores de uma variável latente, superiores às cargas desses mesmos indicadores em variáveis latentes diferentes do modelo.

A Tabela 2 apresenta cargas fatoriais das variáveis latentes, que descrevem de maneira satisfatória os construtos, uma vez que seus valores são mais elevados ao serem associados a outros construtos do modelo da pesquisa. A confiabilidade do modelo testado pode ser conferida pelo valor do peso de cada indicador, que deve ser superior a 0,71. A Tabela 2 demonstra que a maior parte dos pesos dos indicadores apresentou valores superiores a 0,7 , sendo o menor valor identificado relacionado ao indicador ED4 $(0,6231)$ que apresentou valor muito próximo ao parâmetro referenciado.

Tabela 2

Validade discriminante (cargas fatoriais)

\begin{tabular}{ccccccc}
\hline & COMP_USO & INT_USO & EXP_ESF & COND_FAC & EXP_DES & INFL_SOC \\
\hline USO1 & 1,0000 & $-0,2887$ & $-0,2636$ & $-0,3040$ & $-0,3193$ & $-0,3550$ \\
IU1 & $-0,2940$ & 0,9791 & 0,5956 & 0,6223 & 0,6666 & 0,7358 \\
IU2 & $-0,2700$ & 0,9734 & 0,5871 & 0,5803 & 0,6236 & 0,7220 \\
IU3 & $-0,2773$ & 0,9624 & 0,5789 & 0,5485 & 0,6212 & 0,6986 \\
EE1 & $-0,2022$ & 0,4836 & 0,8609 & 0,7265 & 0,6993 & 0,6383 \\
EE2 & $-0,2765$ & 0,6175 & 0,9198 & 0,6126 & 0,6563 & 0,7500 \\
EE3 & $-0,2096$ & 0,5495 & 0,9253 & 0,6322 & 0,6593 & 0,6532 \\
EE4 & $-0,2668$ & 0,5378 & 0,9219 & 0,6220 & 0,6279 & 0,6717 \\
CF1 & $-0,2798$ & 0,5758 & 0,6444 & 0,9479 & 0,9184 & 0,7129 \\
CF2 & $-0,1882$ & 0,4849 & 0,8543 & 0,7299 & 0,6984 & 0,6410 \\
CF3 & $-0,3192$ & 0,5467 & 0,6266 & 0,9375 & 0,8615 & 0,6588 \\
CF4 & $-0,2095$ & 0,4120 & 0,3271 & 0,7243 & 0,5907 & 0,4902 \\
ED1 & $-0,3499$ & 0,6067 & 0,6445 & 0,8259 & 0,8713 & 0,6642 \\
ED2 & $-0,3427$ & 0,5815 & 0,6599 & 0,9527 & 0,9397 & 0,7154 \\
ED3 & $-0,1742$ & 0,6255 & 0,6617 & 0,8176 & 0,9168 & 0,7155 \\
ED4 & $-0,2102$ & 0,3724 & 0,4843 & 0,4590 & 0,6231 & 0,5134 \\
S1 & $-0,3565$ & 0,5966 & 0,4942 & 0,5093 & 0,5631 & 0,8693 \\
IS2 & $-0,3211$ & 0,5871 & 0,5147 & 0,5418 & 0,5917 & 0,8646 \\
IS3 & $-0,1694$ & 0,5801 & 0,8532 & 0,5850 & 0,6035 & 0,7311 \\
IS4 & $-0,2888$ & 0,6084 & 0,5462 & 0,7383 & 0,7263 & 0,7420 \\
\hline
\end{tabular}

Nota. Exp. = Experiência; Gen = Gênero; Id. = Idade; Vol. = Voluntariedade

Fonte: Elaborado pelos autores.

\subsection{Validade Convergente e Confiabilidade de Consistência Interna}

Para Hair et al. (2009), a validade convergente tem como propósito ratificar o quanto a escala está correspondente a outras medidas do conceito - seu critério o valor do AVE (Average Variance Extracted) deve ser maior que 0,50 para cada variável latente. 
No teste, o valor obtido na avaliação convergente foi elevado - acima de 0,5, conforme demonstrado na Tabela 3. O resultado apresentado vem corroborar que os indicadores que mensuram os construtos latentes estão acomodados de forma adequada.

Em relação à confiabilidade de consistência interna, o parâmetro estabelecido é o de que o Alpha de Cronbach $(\geq 0,7)$ indica que os questionamentos componentes de cada construto convergem na direção de mensurar o construto a que pertencem.

Hair et al. (2009) define que a consistência interna é uma média que afere a confiabilidade e robustez existente entre os construtos de uma variável múltipla, onde os indicadores de cada construto devem medir e corresponder ao construto o qual estão relacionados.

A Tabela 3 demonstra a avaliação da confiabilidade de consistência interna do modelo, apresentando vários valores superiores a 0,70 , demonstrando que o resultado apresentado indica que as variáveis latentes apresentam escalas com consistência interna relevante, significando que as afirmativas apresentadas aos respondentes estão adequadamente correspondidas aos seus respectivos construtos, indicando, assim, que o modelo é confiável.

Tabela 3

Variância média extraída e confiabilidade

\begin{tabular}{lccc}
\hline \multicolumn{1}{c}{ Construto } & $\begin{array}{c}\text { Variância } \\
\text { Média Extraída }\end{array}$ & $\mathbf{R}^{\mathbf{2}}$ & Alfa de Chronbach \\
\hline Comportamento de Uso & - & 0,2804 & - \\
Condições Facilitadoras & 0,7087 & 0,0000 & 0,8577 \\
Expectativa de Desempenho & 0,7177 & 0,5307 & 0,8608 \\
Expectativa de Esforço & 0,8233 & 0,0000 & 0,9281 \\
Influência Social & 0,6471 & 0,0000 & 0,8148 \\
Intenção de Uso & 0,9441 & 0,5928 & 0,9704 \\
\hline
\end{tabular}

Nota. Fonte: Elaborado pelos autores.

Após a avaliação dos $\mathrm{R}^{2}$ das variáveis latentes, constata-se a qualidade do modelo, conforme indicado na Tabela 3, que o construto "Intenção Comportamental de Uso", considerado o efeito direto, apresenta o valor de $R^{2}=0,593$ ou, aproximadamente, $59,3 \%$, já quando avaliamos o $R^{2}$ do modelo, considerando o efeito direto e interações, o $R^{2}=0,613$ ou $61,3 \%$ apresenta valores superiores aos resultados obtidos por Venkatesh et al. (2003).

Em seu estudo original, os autores obtiveram o $\mathrm{R}^{2}$ em torno de $40 \%$ e $51 \%$, considerando efeito direto e interações, respectivamente, na análise do construto "Intenção Comportamental" (Venkatesh et al., 2003). A qualidade do modelo também é confirmada pela análise do construto “Comportamento de Uso", que, neste estudo, apresentou $R^{2}=0,280$ para efeitos diretos e $R^{2}=0,330$ para efeitos diretos e interações, ou seja, 28,0 e 33,0\%.

Tabela 4

Cargas do modelo de medidas

\begin{tabular}{lcll}
\hline & Média & Desvio Padrão & Estatística T \\
\hline IU1 <- INT_USO & 0,35138 & 0,00545 & 64,49671 \\
IU2 <- INT_USO & 0,34184 & 0,005548 & 61,60017 \\
IU3 <- INT-USO & 0,33588 & 0,005645 & 59,47677 \\
EE1 <- EXP_ESF & 0,27185 & 0,012654 & 21,50485 \\
EE2 <- EXP-ESF & 0,28936 & 0,00905 & 31,92813 \\
EE3 <- EXP_ESF & 0,27577 & 0,009265 & 29,7605 \\
EE4 <- EXP-ESF & 0,26541 & 0,009545 & 27,82349 \\
CF1 <- COND_FAC & 0,32613 & 0,043147 & 7,597037 \\
CF2 <- COND_FAC & 0,2174 & 0,03793 & 5,813134 \\
CF3 <- COND_FAC & 0,37446 & 0,040936 & 9,135092 \\
CF4 <- COND_FAC & 0,24673 & 0,039386 & 6,231465 \\
ED1 <- EXP_DES & 0,3152 & 0,010882 & 28,92351 \\
ED2 <- EXP_DES & 0,31321 & 0,009287 & 33,68691 \\
ED3 <- EXP_DES & 0,32386 & 0,010315 & 31,38691 \\
ED4 <- EXP_DES & 0,21561 & 0,015791 & 13,72028 \\
IS1 <- INFL_SOC & 0,31302 & 0,016067 & 19,52576 \\
IS2 <- INFL_SOC & 0,30792 & 0,015712 & 19,64943 \\
IS3 <- INFL_SOC & 0,30608 & 0,021079 & 14,47057 \\
IS4 <- INFL_SOC & 0,32028 & 0,023929 & 13,36896 \\
\hline
\end{tabular}

Nota. Fonte: Elaborado pelos autores. 
A obtenção das cargas do modelo de medidas foi realizada por meio de algoritmo do PLS, e a avaliação da significância foi realizada pela técnica Bootstraping, em que foram realizados 1.000 subamostras com reposição, conforme Venkatesh et al. (2003) e podem ser visualizadas na Tabela 4. Nota-se que todas as cargas apresentaram valores significantes.

Na Tabela 5 é possível obervar a capacidade preditiva dos construtos "Expectativa de Desempenho" e "Influência Social" sobre a variável dependente "Intenção Comportamental de Uso", apresentando coeficientes 0,161 e 0,552, valores significantes que evidenciam que as hipóteses H1 e H3 estão suportadas.

Tabela 5

Coeficientes do modelo estrutural, Interações e Significâncias

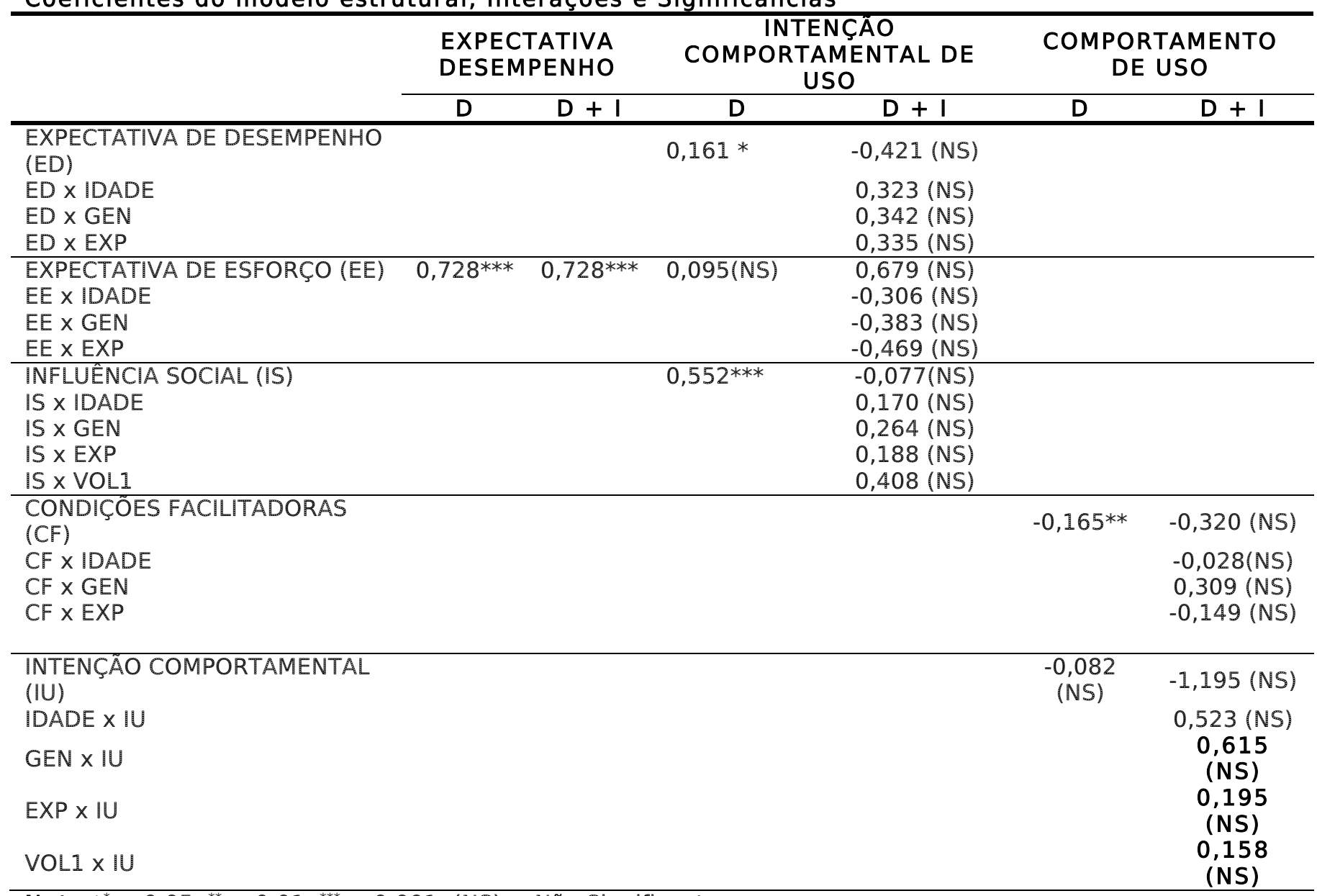

Nota: : $\mathrm{p}<0.05 ;{ }^{* *} \mathrm{p}<0.01 ;{ }^{* * *} \mathrm{p}<0.001 ;(\mathrm{NS})=$ Não Significante

Fonte: Elaborado pelos autores.

De acordo com estes resultados, é possível evidenciar que as influências sociais exercem maior impacto na intenção de uso do sistema do que as percepções de condições facilitadoras, de modo que práticas de divulgação do portal Inovação pelos gestores que promovam o compartilhamento de informações sobre os recursos e funcionalidades potencializam a adoção deste sistema de informação entre os usuários.

Neste sentido, entende-se que a priorização de ações gerenciais que socializem as informações dos benefícios e funcionalidades entre os usuários podem surtir efeitos positivos ao uso regular deste sistema comparada às ações técnicas que envolvam a manipulação de interfaces que ampliem o desempenho do sistema.

Os resultados também evidenciam que os coeficientes obtidos na avaliação dos fatores moderadores "Idade", "Gênero", "Experiência" e "Voluntariedade" na relação entre os construtos "Influência Social", "Condições Facilitadoras" e "Intenção Comportamental de Uso" não foram significantes.

Tais evidências sugerem que campanhas de promoção da adoção deste sistema que explorem as características demográficas individuais podem não obter resultados positivos para o aumento da utilização deste sistema entre os usuários. 
Além disto, infere-se que os resultados obtidos sobre a moderação da experiência individual e idade também possam estar associados à ausência de significância na influência da expectativa de esforço dos usuários, uma vez que estas características dos usuários não manifestaram impactos significantes aos construtos relacionados e os usuários possam desconsiderar eventuais dificuldades na utilização do sistema como um fator impeditivo ao uso do portal.

A seguir, apresenta-se uma síntese da análise das hipóteses suportadas e não suportadas pelo modelo (Figura 4):

\begin{tabular}{clc}
\hline Hipótese & \multicolumn{1}{c}{ Descrição } & Resultado \\
\hline H1 & $\begin{array}{l}\text { A Expectativa de Desempenho influência de forma positiva a Intenção de } \\
\text { Uso do Portal Inovação. }\end{array}$ & Suportado \\
\hline H2 & $\begin{array}{l}\text { A Expectativa de Esforço influência de forma positiva a Intenção de Uso } \\
\text { do Portal Inovação. }\end{array}$ & $\begin{array}{c}\text { Não } \\
\text { Suportado }\end{array}$ \\
\hline H3 & $\begin{array}{l}\text { A Influência Social afeta positiva e intensamente a Intenção de Uso do } \\
\text { Portal Inovação. Dada uma maior pressão social, maior também será a } \\
\text { Intenção de Uso. }\end{array}$ & Suportado \\
\hline H4 & $\begin{array}{l}\text { O impacto das Condições Facilitadoras sobre o Comportamento de Uso } \\
\text { do Portal Inovação será positivo. }\end{array}$ & Suportado \\
\hline H5 & $\begin{array}{l}\text { O impacto da Intenção Comportamental de Uso sobre o Comportamento } \\
\text { de Uso do Portal Inovação será positivo. }\end{array}$ & $\begin{array}{c}\text { Não } \\
\text { Suportado }\end{array}$ \\
\hline
\end{tabular}

Figura 4 - Síntese do resultado das hipóteses

Nota. Fonte: Elaborado pelos autores

\section{Considerações Finais}

A concepção, desenvolvimento e implantação do Portal Inovação coincidem com uma nova configuração que a administração pública tem adotado para atender às demandas da sociedade na atualidade, a qual tem requerido uma maior transparência e efetividade dos serviços públicos. Tais ações denominadas como e-Gov, também coincidem com uma nova configuração político-social da sociedade, que é denominada por Sodré (2007), de ethos midiatizado.

Consequentemente, tais transformações impuseram à administração pública a demanda de maior aproximação com o cidadão, oferecendo uma ampla gama de serviços públicos que atendessem aos novos anseios e necessidades sociais.

Não obstante a isso, o País vivencia um momento muito importante e peculiar no que se refere ao processo de fortalecimento e robustez do SNCTI em resposta aos desafios impostos pelas transformações ocorridas. Sobre isso, nas últimas décadas, o País, sistematicamente, tem desenvolvido políticas públicas de apoio à ciência e à tecnologia, visando o fortalecimento das instituições pertencentes ao SNCTI.

Ao exposto, o desenvolvimento da pesquisa contempla a análise da aceitação do Portal Inovação, identificando os fatores preditivos da Intenção comportamental de Uso e do Comportamento de Uso direcionadores da adoção da tecnologia por seus usuários a partir da extensão do modelo Unificado da Teoria de Aceitação de Tecnologia (UTAUT) e foram obtidos valores de explicação superiores aos estudos realizados por Venkatesh et al. (2003) para o construto "Intenção Comportamental de Uso", seus efeitos diretos e interações, o que corrobora a utilização deste modelo para análise de adoção deste sistema.

Deste modo, as principais constatações acerca dos resultados obtidos demonstram alta magnitude e significância preditiva sobre a Intenção Comportamental de Uso do Portal pelos fatores: Expectativa de Desempenho e Influência Social. Evidenciando, também, que as Condições Facilitadoras impactam significativamente sobre o Comportamento de Uso dos usuários.

Ao exposto, a conclusão basilar do presente estudo é a de que ao considerarmos a aceitação de um portal governamental em que a adoção é voluntária, o fator social é altamente influente na intenção de uso da tecnologia, bem como os aspectos relacionados à produtividade consequente do usuário e o senso de utilidade; além da facilidade de interação e domínio da ferramenta.

Ademais, os resultados obtidos possibilitam mensurar quais variáveis do modelo possuem maior capacidade de influenciar a intenção de uso e, consequentemente, o próprio comportamento de uso do usuário. O que permitiria aos administradores a realização de simulações e estudos objetivando a elaboração de planos gerenciais, que visem monitorar ou avaliar as ações desenvolvidas no âmbito do portal. E, dessa forma direcionar ações específicas, bem como otimizar os investimentos públicos realizados. 
De acordo com os resultados da pesquisa, evidenciou-se que a influência social exerce maior impacto na intenção de uso do Portal Inovação e, portanto, recomendam-se que ações de divulgação e promoção destes portais sejam implementadas futuramente com o objetivo de disseminar e incentivar o uso desta ferramenta.

Os resultados do estudo também sugerem a adoção de práticas gerenciais direcionadoras do desenvolvimento e aperfeiçoamento do Portal que objetivem aprimorar os serviços prestados pelo Portal através do monitoramento das atividades dos usuários pelos gestores do sistema.

A compreensão dos fatores que influenciam positivamente a adoção de sistemas governamentais possibilita avaliar as percepções dos usuários destes serviços que buscam suprir às demandas da sociedade. Dessa forma, o conhecimento destes fatores de influência no uso das tecnologias possibilita a execução de iniciativas futuras que promovam o governo eletrônico brasileiro.

Os resultados contribuem para as pesquisas de adoção de sistemas de informação, apresentando um modelo que reforça e amplia os estudos anteriores associados ao governo eletrônico em um contexto pouco explorado na literatura.

Entre outras limitações do estudo, considera-se o resultado dos valores obtidos nas análises podem ser diferentes em razão das características de outras tecnologias, além de aspectos individuais e outros contextos de uso dos recursos.

Sugere-se a realização de novas pesquisas que contemplem a adoção de aplicações no próprio Portal Inovação direcionada a grupos de usuários específicos (Agentes de Inovação, ICTI's, UIPD, empresas e grupos de pesquisa) como forma de avaliar eventuais diferenças de aceitação do sistema entre grupos.

\section{Notas}

1. Instituído em 12 dezembro de 1980, o ato Bayh-Dole é considerado como um marco na política norteamericana de estímulo à proteção e comercialização de tecnologias. Essa lei consistiu em atribuir as pequenas empresas e as organizações sem fins lucrativos, incluindo as universidades, a titularidade das patentes de invenções obtidas a partir dos resultados dos programas financiados com recursos governamentais.

\section{Referências}

Agune, R., \& Carlos, J. (2005). Governo eletrônico e novos processos de trabalho. In E. Levy \& P. Drago (Eds.), Gestão Pública no Brasil contemporâneo. São Paulo: Fundap.

Al-Gahtani, S. (2001). The applicability of TAM outside North America: An empirical test in the United Kingdom. Information Resources Management Journal, 14(3), 37-46.

Aldricha, D., Bertota, J. C., \& McClurea, C. R. . (2002). E-Government: initiatives, developments, and issues. Government Information Quarterly, 19(4), 349-355.

Barbosa, A. (2008). Governo eletrônico: dimensões da avaliação de desempenho na perspectiva do cidadão. Tese de doutorado, Escola de Administração da Fundação Getulio Vargas, São Paulo, SP, Brasil.

Brasil, Ministério da Ciência, Tecnologia e Inovação (MCTI). Estratégia Nacional de Ciência, Tecnologia e Inovação 2012 - 2015. Balanço das atividades estruturantes, 2011. Recuperado de: http://livroaberto.ibict.br/docs/218981.pdf. Acesso em: 18 jul. 2014.

Castells, M. . (2000). A sociedade em rede: a era da informação: economia, sociedade e cultura (Vol. 1). São Paulo: Paz e Terra.

Cetic.BR. (2011). Governo eletrônico: novas perspectivas para cidadãos e empresas. Retrieved from http://cgi.br/publicacoes/revista/edicao04/cgibr-revistabr-ed4.pdf.

Chiu, C.-M., \& Wang, E. T. G. . (2008). Understanding Web-based learning continuance intention: the role of subjective task value. Information and Management, 45(3), 2008, 194-201.

Correa, D. O. (2011). e-Gov: planejamento de serviços eletrônicos de governo usando mídias cruzadas. Dissertação de Mestrado, Escola Politécnica da Universidade de São Paulo, Departamento de Engenharia de Computação e Sistemas Digitais, São Paulo, SP. 
Damian, I. P. M., \& Merlo, E. M. (2013). Uma análise dos sites de governos eletrônicos no Brasil sob a ótica dos usuários dos serviços e sua satisfação. Revista de Administração Pública, 47(4), 877-899.

Davis, F. D., Bagozzi, R. P., \& Warshaw, P. R. (1989). User Acceptance of Computer Technology: a comparison of two theoretical models. Management Science, 35(8), 982-1003.

Fishbein, M., \& Ajzen, I. (1975). Belief, Attitude, Intention, and Behavior: An Introduction to Theory and Research. Reading, MA: Addison-Wesley.

Fornell, C., \& Lacker, D. F. (1981). Evaluating structural equation models with unobservable variables and measurement error. Journal of Marketing Research, 18(1), 39-50.

Freeman, C. (1995). The national system of innovation in historical perspective. Cambridge Journal of Economics, 19(1), 5-24.

Hair, J. F., Black, W. C. , Babin, B. J., Anderson, R. E., \& Tathan, R.L. (2009). Análise multivariada de dados (6 ed.). Porto Alegre: Bookman.

Koh, C. E. , Ryan, S., \& Prybutok, V. R. (2005). Creating value through managing knowledge in an e-Government to constituency (G2C) environment. The Journal of Computer Information Systems, 45(4), 32-41.

Maciel, C. (2008). Um método para mensurar o grau de maturidae na tomada de decisão edemocrática. Tese de Doutorado, Universidade Federal Fluminense, Rio de Janeiro, RJ.

McNeal, R. S., Tolbert, C. J., Mossberger, K., \& and Dotterweich, L. J. (2003). Innovating in digital government in the American states. Social Science Quarterly, 84(1), 52-70.

OCDE. (1996). Programme On National Innovation Systems. Paper presented at the Industrial competitiveness, Paris.

Osborne, D. (1997). Banishing bureaucracy: the five strategies for reinventing government. New York: Plume.

Prado, E. P. V., Ramalho, N. C. L., Souza, C. A., Cunha, M. A., \& Reinhard, N. (2011). Iniciativas de governo eletrônico: análise das relações entre nível de governo e características dos projetos em casos de sucesso. Resi: Revista Eletrônica de Sistemas de Informação, 10(1), 1-22.

Richardson, R. J. (1989). Pesquisa social: métodos e técnicas (Vol. 2). São Paulo: Atlas.

Ringle, C. M., Wende, S., \& Will, A. (2005). SmartPLS 2.0 M3 (beta). Germany: University of Hamburg. Recuperado de: <http://www.smartpls.de>. Acesso em: 28/07/2009.

Roesch, S. M. A. . (1999). Projetos de estágio e de pesquisa em administração: guias para estágios, trabalhos de conclusão, dissertações e estudo de casos. São Paulo: Atlas.

Sodré, M. (2007). O ethos midiatizado. In Sodré, M. Antropológica do Espelho: uma teoria da comunicação linear e em rede. Rio de Janeiro: Vozes.

Venkatesh, V., Morris, M. G., Davis, G. B., \& Davis, F. D. (2003). User Acceptance of Information Technology: Toward a Unified View. MIS Quarterly, 27(3), 425-478.

Venkatesh, V., \& Davis, F. D. (2000). A Theoretical Extension of the Technology Acceptance Model: Four Longitudinal Field Studies. Management Science, 46(2), 186-204.

Yurong, Y., \& Murphy, L. (2007). Remote electronic voting systems: an exploration of voters perceptions and intention to use. European Journal of Information Systems, 16, 106-120. 


\section{Apêndice}

Questionário do Estudo

\begin{tabular}{|c|c|}
\hline \multicolumn{2}{|c|}{ Expectativa de Desempenho } \\
\hline ED1 & Eu acho que o Portal Inovação é útil em meu trabalho. \\
\hline ED2 & O uso do Portal Inovação permite-me realizar minhas atividades mais rapidamente. \\
\hline ED3 & O uso do Portal Inovação aumentará minha produtividade. \\
\hline ED4 & Utilizando o Portal Inovação terei mais chances de ter um aumento de salarial. \\
\hline \multicolumn{2}{|c|}{ Expectativa de Esforço } \\
\hline EE1 & Minha interação com o Portal Inovação é clara e de fácil compreensão. \\
\hline EE2 & Será fácil tornar-me um hábil usuário do Portal Inovação. \\
\hline EE3 & Eu acho que o Portal Inovação será fácil de utilizar. \\
\hline EE4 & Aprender a utilizar o Portal Inovação é (foi) fácil para mim. \\
\hline \multicolumn{2}{|c|}{ Influência Social } \\
\hline IS1 & As pessoas com quem me relaciono acham que eu deveria utilizar o Portal Inovação. \\
\hline IS2 & As pessoas que são importantes para mim acham que eu deveria utilizar o Portal Inovação. \\
\hline IS3 & A gerência e direção da empresa têm me dado suporte para utilizar o Portal Inovação. \\
\hline IS4 & Em geral, a organização que atuo tem incentivado a utilização do Portal Inovação. \\
\hline \multicolumn{2}{|c|}{ Condições Facilitadoras } \\
\hline CF1 & $\begin{array}{l}\text { Eu tenho os recursos (tempo, computador, acesso à Internet etc.) necessários para utilizar o } \\
\text { Portal Inovação. }\end{array}$ \\
\hline CF2 & Eu tenho o(s) conhecimento(s) necessário(s) para utilizar o Portal Inovação. \\
\hline CF3 & O Portal Inovação é compatível com outros sistemas que utilizo. \\
\hline CF4 & O Portal Inovação oferece auxílio adequado às dificuldades de uso. \\
\hline \multicolumn{2}{|c|}{ Intenção de Uso } \\
\hline IU1 & Eu pretendo utilizar o Portal Inovação nos próximos meses. \\
\hline IU2 & Eu creio que utilizarei o Portal Inovação nos próximos meses. \\
\hline IU3 & Eu planejo utilizar o Portal Inovação nos próximos meses. \\
\hline \multicolumn{2}{|c|}{ Comportamento de Uso } \\
\hline USO1 & Quantas vezes em média você utilizou o Portal Inovação nos últimos meses? $(\mathrm{xx})$ vezes no mês. \\
\hline \multicolumn{2}{|c|}{ Variáveis Moderadoras } \\
\hline GEN & Sexo: \\
\hline IDADE & Qual sua idade? \\
\hline EXP & Há quanto tempo você é usuário do Portal Inovação? \\
\hline VOL1 & Ascrever quantos açaño em que atua requer que você utilize o Portal Inovação para a realização de suas \\
\hline \multicolumn{2}{|c|}{ Questões de Controle } \\
\hline CIVIL & Qual é o seu estado civil? \\
\hline SETOR & A qual setor você está vinculado? \\
\hline ESCO & Qual é o seu nível de escolaridade? \\
\hline REGIAO & Em qual região você atua? \\
\hline
\end{tabular}

